\title{
EDITORIAL
}

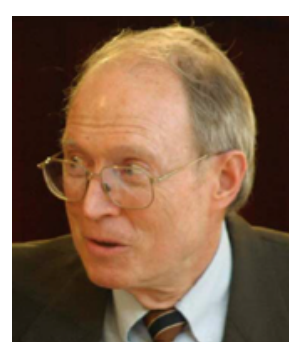

\section{Letter of Congratulations on the Publication of Biomedical Engineering Letters}

\author{
John G. Webster
}

(C) The Korean Society of Medical \& Biological Engineering and Springer 2011

I congratulate you on the publication of Biomedical Engineering Letters. I have read through the first inaugural issue and find it represents the kind of biomedical engineering that interests me. The review article Recent Research Trends in Nanoscale Electro-mechanical Systems for Bio-medical Applications described electrical recording of the cell potential from a beating cardiomyocyte using a bioprobe with the nanoFET. It also described the problems with interference by noise. The review article Monitoring Physiological Signals Using Nonintrusive Sensors Installed in Daily Life Equipment included useful information to me on the use of capacitively coupled electrodes to measure the ECG without contact to the body. The review article Mitochondrial Medicine and Biomedical Engineering described the way mitrochondia are closely related to the macroscopic energy balance of the human body. The original article Development of FPGAbased Coincidence Units with Veto Function described a field programmable gated array (FPGA)-based coincidence detector for positron emission tomography (PET). The original article An Improved Technique to Consider Mismatches between fMRI and EEG/MEG Sources for fMRI Constrained EEG/MEG Source Imaging addresses the problem that fMRI has slow temporal resolution compared to the fast EEG/ MEG temporal resolution. The original article Characteristics of PLLA Films Blended with PEG Block Copolymers as Additives for Biodegradable Polymer Stents found that PLLA films blended with hydrophilic PEG-based block copolymer as additives demonstrated improved swelling property as well as mechanical properties. The original article
Compression Induced Contrast Change in X-ray Mammograms: A Simulation Study on breast electrography found that breast compression may be useful to detect the harder breast tumors. The original article In Vivo Magnetic Resonance Electrical Impedance Tomography of Canine Brain: Disease Model Study of Ischemia and Abscess describes how injecting $5 \mathrm{~mA}$ of electric current when using MRI electrical impedance tomography produces a conductivity contrast corresponding to brain ischemia and abscess. The original article A New Approach to Ophthalmic Imaging with Concave Array Transducers describes how using a concave array in ultrasonic imaging yields high-spatial, high-quality ophthalmic images.

These articles should assist biomedical engineers who are developing electrical and mechanical sensors to solve important medical problems such as to noninvasively measure intracranial pressure to monitor hydrocephalus, to monitor the elderly who remain in their homes, etc. These articles should assist biomedical engineers who are developing chemical sensors to solve important medical problems such as implantable glucose sensors to develop a closed loop system to treat diabetes, etc. These articles should also assist biomedical engineers who are developing therapeutic medical devices such as cardiac and liver ablation to destroy tissue such as cancer. I look forward to reading more future articles that inform biomedical engineers of the latest information that will help them in their development of diagnostic and therapeutic methods and equipment to contribute to improved health care.

John G. Webster $(\varangle)$

Department of Biomedical Engineering, College of Engineering, University of Wisconsin-Madison 2148 Engineering Centers Building 1550 Engineering Drive Madison, WI 53706, USA

Tel : +1-608-263-1574; Fax : +1-608-265-9239

E-mail : webster@engr.wisc.edu 